PROCEEDINGS OF THE

AMERICAN MATHEMATICAL SOCIETY

Volume 133, Number 9, Pages 2501-2505

S 0002-9939(05)07429-0

Article electronically published on April 19, 2005

\title{
A PURELY ALGEBRAIC CHARACTERIZATION OF THE HYPERREAL NUMBERS
}

\author{
VIERI BENCI AND MAURO DI NASSO
}

(Communicated by Carl G. Jockusch, Jr.)

\begin{abstract}
The hyperreal numbers of nonstandard analysis are characterized in purely algebraic terms as homomorphic images of a suitable class of rings of functions.
\end{abstract}

\section{INTRODUCTION}

Since the seminal classical work by E. Hewitt [7] appeared over sixty years ago, the algebraic/topological study of rings of functions has been constantly alive in the literature (see e.g. [6], [1, [10, 4] and [9]). Recently, in their book 5], G. Dales and H. Woodin gave new insights to the subject by deeply investigating a class of totally ordered real fields, namely the superreal fields. Among them, the so-called hyperreal fields and the ultrapowers. Now, all ultrapowers are hyperreals ${ }^{*} \mathbb{R}$ of nonstandard analysis (nonstandard reals), but the two notions of hyperreal fields are different.

The very definition of nonstandard reals $* \mathbb{R}$, as usually given in the literature, requires notions from mathematical logic. Precisely, such definition is formulated by means of the Leibniz transfer principle, an elementary embedding property for bounded quantifier formulas in the language of set theory.

The goal of this paper is to provide an alternative equivalent definition of $* \mathbb{R}$ in purely algebraic (and elementary) terms. Precisely, we shall characterize the hyperreal fields of nonstandard analysis as homomorphic images of composable rings $\mathcal{F}$ of real-valued functions ("composable" means closed under compositions with any function $f: \mathbb{R} \rightarrow \mathbb{R}$ ).

From a philosophical point of view, our proposed definition of ${ }^{*} \mathbb{R}$ could be justified by the following facts:

- The operations on $\mathcal{F}$ are defined point-wise, hence the operations on ${ }^{*} \mathbb{R}$ are directly inherited from the usual field operations on $\mathbb{R}$.

- A crucial feature of the nonstandard real numbers ${ }^{*} \mathbb{R}$ is that every function $f: \mathbb{R} \rightarrow \mathbb{R}$ has a nonstandard extension ${ }^{*} f:{ }^{*} \mathbb{R} \rightarrow{ }^{*} \mathbb{R}$ that satisfies the same "elementary" properties. Thanks to composability, the nonstandard extension ${ }^{*} f$ can be defined in a natural way, by means of its natural "lifting" $\widehat{f}: \mathcal{F} \rightarrow \mathcal{F}$ given by $\widehat{f}(\varphi)=f \circ \varphi$.

Received by the editors November 13, 2002 and, in revised form, July 11, 2003.

2000 Mathematics Subject Classification. Primary 16S60, 54C40, 26E35.

Key words and phrases. Rings of functions, algebraic properties of functions spaces, nonstandard analysis. 
- There is no need to postulate the Leibniz transfer principle, because that logical principle follows from our definition.

As a side result, this definition makes it possible to naturally accomodate the nonstandard reals in the Dales-Woodin's algebraic hierarchy of superreal fields [5].

\section{THE HYPERREAL NUMBERS OF NONSTANDARD ANALYSIS}

For a detailed presentation of the superstructure approach to nonstandard analysis, and for the unexplained notions and notation, we refer to [3], §4.4. For completeness, we briefly recall here the crucial definitions.

Definition 2.1. For any set $X$ of atoms, the superstructure over $X$ is the set $V(X)=\bigcup_{n \in \mathbb{N}} V_{n}(X)$, where $V_{0}(X)=X$ and $V_{n+1}(X)=\mathcal{P}\left(V_{n}(X)\right)$ is the powerset of $V_{n}(X)$. A nonstandard embedding is a mapping $*: V(\mathbb{R}) \rightarrow V\left({ }^{*} \mathbb{R}\right)$ that satisfies the Leibniz transfer principle, i.e. for every bounded quantifier formula $\sigma\left(x_{1}, \ldots, x_{n}\right)$ and elements $a_{1}, \ldots, a_{n} \in V(\mathbb{R}), \sigma\left(a_{1}, \ldots, a_{n}\right) \Leftrightarrow \sigma\left({ }^{*} a_{1}, \ldots,{ }^{*} a_{n}\right)$. It is assumed that ${ }^{*} r=r$ for every $r \in \mathbb{R}$ and that ${ }^{*} \mathbb{R}$ is a set of atoms.

By bounded quantifier formula we mean a first-order formula in the language $\mathcal{L}=\{\in\}$ of set theory, where all quantifiers occur in the bounded forms $\forall x \in y \ldots$ (i.e. $\forall x x \in y \rightarrow \ldots$ ) or $\exists x \in y \ldots$ (i.e. $\exists x x \in y \wedge \ldots$ ). In the literature, nonstandard embeddings also satisfy the condition $\mathbb{N} \neq{ }^{*} \mathbb{N}$, but for simplicity we do not assume it here. In particular, the identity map on $V(\mathbb{R})$ is also allowed as the trivial nonstandard embedding.

Definition 2.2. A field $\mathbb{F}$ is a set of nonstandard reals (or hyperreal numbers of nonstandard analysis) if there exists a nonstandard embedding $*: V(\mathbb{R}) \rightarrow V\left({ }^{*} \mathbb{R}\right)$ where ${ }^{*} \mathbb{R}=\mathbb{F}$.

In [8, H.J. Keisler showed that, up to isomorphisms, the nonstandard reals are precisely the limit ultrapowers of $\mathbb{R} 1$ The characterization theorem we present in the next section could be proved by taking that result as a starting point. However, we prefer to give a direct proof, in order to make this paper self-contained and to keep our treatment as close to the basic language of algebra as possible.

\section{THE CHARACTERIZATION THEOREM}

Definition 3.1. Let $R$ be a given ring. For any set $I$, denote by $R^{I}$ the ring of all functions $\varphi: I \rightarrow R$ where operations are defined pointwise. A ring of (R-valued) functions $\mathcal{F}$ is a subring of some $R^{I}$. It is assumed that a ring of functions contains all constant functions.

The crucial notion we shall use in the sequel is the following.

Definition 3.2. A ring of functions $\mathcal{F} \subseteq R^{I}$ is composable if for every $\varphi \in \mathcal{F}$ and for every $f: R \rightarrow R$, the composition $f \circ \varphi: I \rightarrow R$ is in $\mathcal{F}$.

Rings of the form $R^{I}$ are trivially composable. Other examples are

$$
\mathcal{F}=\left\{\varphi: I \rightarrow R|| \operatorname{ran} \varphi \mid \leq \aleph_{0}\right\},
$$

${ }^{1}$ The limit ultrapowers are a generalization of the ultrapowers. Definitions and basic results can be found in [3], $\S 6.4$. 
the rings of those functions taking at most countably many values. We remark that composability can be seen as a lifting property, because it allows extending each function $f: R \rightarrow R$ to a function $\widehat{f}: \mathcal{F} \rightarrow \mathcal{F}$ by putting $\widehat{f}(\varphi)=f \circ \varphi$.

We are now ready to prove the characterization theorem that we propose as an alternative definition of nonstandard reals.

Theorem 3.3. A field $\mathbb{F}$ is a set of nonstandard reals if and only if it is a homomorphic image of some composable ring $\mathcal{F}$ of real-valued functions.

Proof. Assume first that there is a surjective ring-homomorphism $J: \mathcal{F} \rightarrow \mathbb{F}$ where $\mathcal{F} \subseteq \mathbb{R}^{I}$ is a composable ring of real-valued functions. Without loss of generality we can assume that $J\left(c_{r}\right)=r$ for all $r \in \mathbb{R}$, where $c_{r}$ denotes the constant function with value $r$. We have to show that there is a nonstandard embedding $*: V(\mathbb{R}) \rightarrow V(\mathbb{F})$.

For every $\varphi \in \mathcal{F}$, denote by $Z(\varphi)=\{i \in I \mid \varphi(i)=0\}$ its zero set. Then the family $\{Z(\varphi) \mid J(\varphi)=0\}$ is a filter base that can be extended to an ultrafilter $\mathcal{U}$ on $I$. On the set of functions

$$
\mathcal{G}=\left\{\varphi: I \rightarrow A \mid A \in V(\mathbb{R}) \text { and } \exists \varphi^{\prime} \in \mathcal{F} \exists h \text { with } \varphi=h \circ \varphi^{\prime}\right\}
$$

consider the equivalence relation $\varphi \sim \psi \Leftrightarrow\{i \in I \mid \varphi(i)=\psi(i)\} \in \mathcal{U}$ and the pseudo-membership relation $\psi \triangleleft \varphi \Leftrightarrow\{i \in I \mid \psi(i) \in \varphi(i)\} \in \mathcal{U}$. Then define the mapping $\Psi: \mathcal{G} / \sim \rightarrow V(\mathbb{F})$ by putting

$$
\Psi([\varphi])=J(\vartheta) \quad \text { if } \varphi \sim \vartheta \in \mathcal{F} \quad \text { and } \Psi([\varphi])=\{\Psi([\psi]) \mid \psi \triangleleft \varphi\} \text { otherwise. }
$$

Without loss of generality, we are assuming that $\mathbb{F}$ is a set of atoms. It can be directly verified that the above definition is well posed. The mapping $\Psi$ satisfies the following version of tos theorem. For every $\varphi_{1}, \ldots, \varphi_{n} \in \mathcal{G}$ and for every bounded quantifier formula $\sigma\left(x_{1}, \ldots, x_{n}\right)$,

$$
\sigma\left(\Psi\left(\left[\varphi_{1}\right]\right), \ldots, \Psi\left(\left[\varphi_{n}\right]\right)\right) \Leftrightarrow\left\{i \in I \mid \sigma\left(\varphi_{1}(i), \ldots, \varphi_{n}(i)\right)\right\} \in \mathcal{U} .
$$

The proof is by induction on the complexity of formulas. Everything is straightforward, except one implication at the quantifier step, where the composability property of $\mathcal{F}$ is used in an essential way. Precisely, let $\varphi_{s}^{\prime} \in \mathcal{F}$ and let $\varphi_{s}=h_{s} \circ \varphi_{s}^{\prime} \in \mathcal{G}$ for $s=0, \ldots, n$. Assume that

$$
\Lambda=\left\{i \in I \mid \exists x \in \varphi_{0}(i) \sigma\left(x, \varphi_{1}(i), \ldots, \varphi_{n}(i)\right)\right\} \in \mathcal{U} .
$$

Let $\mathcal{B}$ be a base of $\mathbb{R}$ as a vector space on $\mathbb{Q}$. Since $\mathcal{B}$ has the power of the continuum, we can find 1-1 maps $f_{s}: \mathbb{R} \rightarrow \mathcal{B}$ with pairwise disjoint ranges. By the composability of $\mathcal{F}$, the function $\psi=\left(\sum_{s=0}^{n} f_{s} \circ \varphi_{s}^{\prime}\right) \in \mathcal{F}$. Notice that, by linear independency, $\psi(i)=\psi(j) \Rightarrow\left(f_{s} \circ \varphi_{s}^{\prime}\right)(i)=\left(f_{s} \circ \varphi_{s}^{\prime}\right)(j)$ for all $s \Rightarrow \varphi_{s}^{\prime}(i)=\varphi_{s}^{\prime}(j)$ for all $s$, hence $\varphi_{s}(i)=\varphi_{s}(j)$ for all $s$. In particular, there exists a function $\zeta$ such that:

- For every $i \in \Lambda, \zeta(i) \in \varphi_{0}(i)$ witnesses $\sigma\left(\zeta(i), \varphi_{1}(i), \ldots, \varphi_{n}(i)\right)$,

- $\zeta(i)=\zeta(j)$ whenever $\psi(i)=\psi(j)$.

As a straight consequence of the latter property, there is a function $h$ with $\zeta=h \circ \psi$, hence $\zeta \in \mathcal{G}$. We can now apply the inductive hypothesis and obtain

$$
\Psi([\zeta]) \in \Psi\left(\left[\varphi_{0}\right]\right) \wedge \sigma\left(\Psi([\zeta]), \Psi\left(\left[\varphi_{1}\right]\right), \ldots, \Psi\left(\left[\varphi_{n}\right]\right)\right) .
$$

Now define $*: V(\mathbb{R}) \rightarrow V(\mathbb{F})$ as the mapping where ${ }^{*} r=r$ if $r \in \mathbb{R}$, and ${ }^{*} A=\{\Psi([\varphi]) \mid \varphi: I \rightarrow A\}$ otherwise. The definition is well posed and ${ }^{*} \mathbb{R}=\mathbb{F}$. 
Since $\Psi$ satisfies Los theorem, it is easily seen that the Leibniz transfer principle holds and so $*$ is the desired nonstandard embedding 2

Vice versa, assume that $*: V(\mathbb{R}) \rightarrow V\left({ }^{*} \mathbb{R}\right)$ is a nonstandard embedding. Let $I$ be the set of all finite collections of hyperreal numbers and real functions $f: \mathbb{R}^{k} \rightarrow \mathbb{R}$ (of several variables).

Our next goal is to find a function $\Phi:{ }^{*} \mathbb{R} \rightarrow \mathbb{R}^{I}$ and a maximal ideal $M$ in such a way that the composition $K=\pi \circ \Phi:{ }^{*} \mathbb{R} \rightarrow \mathbb{R}^{I} / M$ is a 1-1 ring-homomorphism $\left(\pi: \mathbb{R}^{I} \rightarrow \mathbb{R}^{I} / M\right.$ is the canonical projection).

We claim that for every $i \in I$, one can find a 1-1 mapping $\chi_{i}:{ }^{*} \mathbb{R} \rightarrow \mathbb{R}$ such that:

(1) $\chi_{i}(r)=r$ for all real numbers $r \in i$;

(2) $\chi_{i}\left({ }^{*} f\left(a_{1}, \ldots, a_{k}\right)\right)=f\left(\chi_{i}\left(a_{1}\right), \ldots, \chi_{i}\left(a_{k}\right)\right)$ for all $k$-variable functions $f \in i$, and for all hyperreals $a_{1}, \ldots, a_{k} \in i$.

Enumerate all the equalities ${ }^{*} f_{j}\left(a_{j 1}, \ldots, a_{j k_{j}}\right)={ }^{*} g_{j}\left(b_{j 1}, \ldots, b_{j h_{j}}\right)$ for $j=1, \ldots, n$, where the functions $f_{j}: \mathbb{R}^{k_{j}} \rightarrow \mathbb{R}$ and $g_{j}: \mathbb{R}^{h_{j}} \rightarrow \mathbb{R}$ are in $i$, and the hyperreal numbers $a_{j l}, b_{j l} \in i$. Then the following bounded formula is true:

$$
\exists x_{11}, \ldots, x_{n k_{n}}, y_{11}, \ldots, y_{n h_{n}} \in{ }^{*} \mathbb{R}\left(\bigwedge_{j=1}^{n} f_{j}\left(x_{j 1}, \ldots, x_{j k_{j}}\right)={ }^{*} g_{j}\left(y_{j 1}, \ldots, y_{j h_{j}}\right)\right) \text {. }
$$

By the Leibniz transfer principle, there are $r_{j l}, s_{j l} \in \mathbb{R}$ that satisfy all the corresponding standard equalities $f_{j}\left(r_{j 1}, \ldots, r_{j k_{j}}\right)=g_{j}\left(s_{j 1}, \ldots, s_{j h_{j}}\right)$ for $j=1, \ldots, n$. Note that, by simple modifications of the above formula, we can assume the following:

(a) $r_{j l}=a_{j l}$ (and $s_{j l}=b_{j l}$ ) whenever $a_{j l} \in \mathbb{R}$ (or $b_{j l} \in \mathbb{R}$, respectively).

(b) $r_{j l}=r_{j^{\prime} l^{\prime}}$ (and $s_{j l}=s_{j^{\prime} l^{\prime}}$ ) whenever $a_{j l}=a_{j^{\prime} l^{\prime}}$ (or $b_{j l}=b_{j^{\prime} l^{\prime}}$, respectively).

(c) $r_{j l}=s_{j^{\prime} l^{\prime}}$ whenever $a_{j l}=b_{j^{\prime} l^{\prime}}$.

Namely, property (a) can be obtained by omitting those existential quantifiers that correspond to real numbers (real numbers are to be taken as parameters). As for (b) and (c), one adds to the formula the conjunction of the corresponding equalities $x_{j l}=x_{j^{\prime} l^{\prime}}, y_{j l}=y_{j^{\prime} l^{\prime}}$, and $x_{j l}=y_{j^{\prime} l^{\prime}}$.

As a consequence of (a), (b) and (c), a 1-1 mapping $\chi_{i}:{ }^{*} \mathbb{R} \rightarrow \mathbb{R}$ can be defined in such a way that $\chi_{i}\left(a_{j l}\right)=r_{j l}$ and $\chi_{i}\left(b_{j l}\right)=s_{j l}$, and the required properties (1) and (2) are fulfilled.

Now define $\Phi:{ }^{*} \mathbb{R} \rightarrow \mathbb{R}^{I}$ by putting $\Phi(a)=\Phi_{a}$, where $\Phi_{a}(i)=\chi_{i}(a)$ for all $i \in I$. By condition $(1), \Phi(r)=c_{r}$ for every $r \in \mathbb{R}$.

For any given $a, b \in * \mathbb{R}$, let $j(a, b) \in I$ be the finite collection which consists of $a, b$ and of the sum and product functions $+, \cdot: \mathbb{R}^{2} \rightarrow \mathbb{R}$.

By condition (2), for all $i \supseteq j(a, b)$ :

- $\Phi_{a}(i)+\Phi_{b}(i)=\chi_{i}(a)+\chi_{i}(b)=\chi_{i}(a+b)=\Phi_{a+b}(i)$;

- $\Phi_{a}(i) \cdot \Phi_{b}(i)=\chi_{i}(a) \cdot \chi_{i}(b)=\chi_{i}(a \cdot b)=\Phi_{a \cdot b}(i)$.

In particular, for all $a, b \in{ }^{*} \mathbb{R}$, both $\Phi_{a}+\Phi_{b}-\Phi_{a+b}$ and $\Phi_{a} \cdot \Phi_{b}-\Phi_{a \cdot b}$ belong to the following ideal:

$$
P=\left\{\varphi \in \mathbb{R}^{I} \mid \exists j \in I \text { such that } \varphi(i)=0 \text { for all } i \supseteq j\right\} .
$$

\footnotetext{
${ }^{2}$ The particular case $\mathcal{F}=\mathbb{R}^{I}$ of this implication was treated in [2].
} 
Now pick $M$ any maximal ideal extending $P$, and let $\pi: \mathbb{R}^{I} \rightarrow \mathbb{R}^{I} / M$ be the canonical projection onto the corresponding quotient field. Then the composition $K=\pi \circ \Phi:{ }^{*} \mathbb{R} \rightarrow \mathbb{R}^{I} / M$ is a ring-homomorphism because $K(a)+K(b)-K(a+b)=0$ and $K(a) \cdot K(b)-K(a \cdot b)=0$ for all $a, b \in{ }^{*} \mathbb{R}$. Note that $K$ is necessarily 1-1 since all $\chi_{i}$ are. In order to get an isomorphism out of $K$, consider the following family of functions:

$$
\begin{aligned}
\mathcal{F}= & \left\{\varphi \in \mathbb{R}^{I} \mid \exists a_{1}, \ldots, a_{n} \in{ }^{*} \mathbb{R}\right. \text { such that } \\
& \text { if } \left.\chi_{i}\left(a_{s}\right)=\chi_{j}\left(a_{s}\right) \text { for all } s=1, \ldots, n \text { then } \varphi(i)=\varphi(j)\right\} .
\end{aligned}
$$

A straightforward verification proves that $\mathcal{F}$ is a composable subring of $\mathbb{R}^{I}$. Since trivially $\operatorname{ran} \Phi \subseteq \mathcal{F}$, it makes sense to consider the composition

$$
K^{\prime}=\pi^{\prime} \circ \Phi:{ }^{*} \mathbb{R} \rightarrow \mathbb{F},
$$

where $\pi^{\prime}: \mathcal{F} \rightarrow \mathbb{F}=\mathcal{F} / M^{\prime}$ is the restriction of $\pi$ that projects $\mathcal{F}$ onto its quotient field modulo $M^{\prime}=M \cap \mathcal{F}$. $K^{\prime}$ is a 1-1 ring-homomorphism because $K$ is.

In order to prove that $K$ is an isomorphism, we are left to show that for every $\varphi \in \mathcal{F}$, there exists $b \in{ }^{*} \mathbb{R}$ with $\pi(\varphi)=\pi\left(\Phi_{b}\right)$. By the definition of $\mathcal{F}$, there are finitely many hyperreals $a_{1}, \ldots, a_{n}$ such that $\varphi(i)=\varphi(j)$ whenever $\chi_{i}\left(a_{s}\right)=\chi_{j}\left(a_{s}\right)$ for all $s=1, \ldots, n$. But then we can pick a function $f: \mathbb{R}^{n} \rightarrow \mathbb{R}$ such that $f\left(\chi_{i}\left(a_{1}\right), \ldots, \chi_{i}\left(a_{n}\right)\right)=\varphi(i)$ for all $i \in I$. If $b={ }^{*} f\left(a_{1}, \ldots, a_{n}\right)$, by the condition (2) above, $\Phi_{b}(i)=\varphi(i)$ for all $i \supseteq\left\{a_{1}, \ldots, a_{n}, f\right\} \in I$, hence $\pi\left(\Phi_{b}\right)=\pi(\varphi)$ as desired. The composition $J=K^{\prime-1} \circ \pi^{\prime}: \mathcal{F} \rightarrow * \mathbb{R}$ is the surjective ring-homomorphism we were looking for.

\section{ACKNowledgement}

The authors thank Marco Forti for many valuable discussions.

\section{REFERENCES}

[1] M.Y. Antonovskij, D.V. Chudnovsky, G.V. Chudnovsky and E. Hewitt, Rings of real-valued continuous functions. II, Math. Z. 176 (1981), 151-186. MR0607959 (83f:54011)

[2] V. Benci and M. Di Nasso, A ring homomorphism is enough to get nonstandard analysis, Bull. Belg. Math. Soc. - S. Stevin 10 (2003), 481-490. MR2040525 (2004k:16084)

[3] C.C. Chang and H.J. Keisler, Model Theory (3rd edition), North-Holland, 1990. MR1059055 (91c:03026)

[4] G.L. Cherlin and M.A. Dickmann, Real closed rings I. Residue rings of rings of continuous functions, Fund. Math. 126 (1986), 147-183. MRR0843243 (87h:12001)

[5] H.G. Dales and W.H. Woodin, Super-real Fields, Oxford University Press, 1996. MR1420859 (98b:12005)

[6] L. Gillman and M. Jerison, Rings of Continuous Functions, Van Nostrand, 1960. MR0116199 (22:6994)

[7] E. Hewitt, Rings of real-valued continuous functions, Trans. Amer. Math. Soc. 64 (1948), 45-99. MR 0026239 (10,126e)

[8] H.J. Keisler, Foundations of Infinitesimal Calculus, Prindle, Weber \& Schmidt, 1976.

[9] J.J. Moloney, Residue class domains of the ring of convergent sequences and of $C^{\infty}([0,1], \mathbb{R})$, Pacific J. Math. 143 (1990), 79-153. MR1047403 (91k:12009)

[10] J. Roitman, Non-isomorphic hyper-real fields from non-isomorphic ultrapowers, Math. Z. 181 (1982), 93-96. MR0671717(84a:54030)

Dipartimento di Matematica Applicata "Ulisse Dini", Università di Pisa, Pisa, Italy

E-mail address: benci@dma.unipi.it

Dipartimento di Matematica "Leonida Tonelli", Università di Pisa, Pisa, Italy

E-mail address: dinasso@dm.unipi.it 